\section{JTI}

JOURNAL OF

TRAUMA AND INJURY

\title{
Vascular Injuries Due to Penetrating Missile Trauma in Anti-Terrorism Ops
}

\author{
Rishi Dhillan, M.B.B.S., M.S., D.N.B., F.A.C.S., Alok Bhalla, M.B.B.S., M.S., M.Ch., \\ Sushil Kumar Jha, M.B.B.S., M.S., M.Ch., Hakam Singh, M.B.B.S., M.S., \\ Aman Arora, M.B.B.S., M.S., D.N.B.
}

Department of Surgery, Base Hospital, Srinagar, India

Received: August 7, 2018

Revised: October 9, 2018

Accepted: October 18, 2018

\section{Correspondence to}

Rishi Dhillan, M.B.B.S., M.S.,

\section{D.N.B., F.A.C.S.}

Department of Surgery, Base Hospital, H-108, B Building, Ajnara Pride, Sector 4B, Vasundhara, Ghaziabad, Uttar Pradesh 201010, India

Tel: +91-8130294270, +91-9419864334

Fax: +91-1942706727

E-mail: rishidhillan@gmail.com

Purpose: Penetrating vascular trauma though less common poses a challenge to all Surgeons. This study was designed to analyse the profile, management modalities of vascular trauma and the outcomes thereof at a Trauma Care Centre in a Tertiary care setting in hostile environment in India.

Methods: A prospective review of all patients with arterial and venous injuries being transferred to the Trauma Center at out Tertiary Care Center between June 2015 and May 2018 was done. Demographics, admission data, treatment, and complications were reviewed.

Results: There were a total of 46 patients with 65 vascular injuries, 39 arterial injuries and 26 venous injuries. The age range was 21 to 47 years. Nineteen patients had both arterial and venous injuries. A total of 42 cases presented within 12 hours of injury and complete arterial transections were found in 33 cases (80.49\%). There were three mortalities (6.52\%) and three amputations (8.33\%). The overall limb salvage rate was $91.67 \%$ with popliteal artery being the commonest injured artery. Poor prognosticators for limb salvage were increasing time to present to the trauma centre, hypovolemic shock, multi-organ trauma and associated venous injuries.

Conclusions: Penetrating missile trauma leading to vascular injuries has not been widely reported. Attempting limb salvage even in cases with delayed presentation should be weighed with the threat to life before revascularisation and should preferably be done at a centre with vascular expertise. A team approach with vascular, orthopaedic, general surgeons, and critical care anaesthesiologists all aboard improve the outcomes manifold. Use of tourniquets and early fasciotomies have been emphasized as is the use of native veins as the bypass conduit. This is probably the largest study on penetrating Vascular trauma in anti-terrorism ops from the Indian subcontinent. It highlights the significance of prompt recognition and availability of vascular expertise in optimally managing cases of vascular trauma.

Keywords: Penetrating vascular trauma; Blast injuries; Trauma center; Fasciotomy; Vascular trauma terrorism 


\section{INTRODUCTION}

Traumatic vascular injuries are infrequent but can be devastating [1]. Vascular trauma poses a challenge to general and trauma surgeons faced by such situations, especially cases of polytrauma. Prompt recognition is the key to optimum management for arterial and venous injuries. Outcomes pertaining to mortality and limb salvage are bleak in the vast Indian subcontinent with delayed presentation and prolonged transfer time periods. This study was designed to analyse the profile and management modalities of vascular trauma and the outcomes thereof at a trauma care centre in a tertiary care setting in hostile environment. The objective of this study was to analyze the clinical presentation of arterial and venous injuries in the scenario of anti-terrorist action and to study the profile of patients and type of trauma suffered that leads to arterial and venous trauma in our setting. We attempted to critically analyze the decisions, methods of repair and limb salvage rates and studied the post treatment morbidity and mortality in all patients.

\section{METHODS}

This was a prospective study on all patients with arterial and venous injuries suffered during anti-terrorist action presenting to Base Hospital, Srinagar, Jammu and Kashmir, India. Hospital ethical committee clearance was obtained prior to commencing the study and written informed consent was taken from all patients except those in shock, where consent was taken from persons accompanying them. The injuries suffered were mostly due to gunshot wounds or blast injuries from grenades or anti-personnel mines. The study was done over a period of 3 years from June 2015 to May 2018. Demographics, admission data and outcomes were reviewed. Follow-up ranged from 1 month to 2 years. Inclusion criteria were all patients reporting with arterial and venous trauma. Patients who were brought in dead were excluded. The parameters noted were age of the patient, sex of the patient, duration from time of trauma till presentation and time till definitive intervention, associated injuries, type of trauma leading to the injury, presence or absence of hypotension, partial or complete injury, ankle brachial index (ABI) for lower limb injuries and pressures at wrist for upper limb injuries. It was noted whether a tourniquet was in place as the patient reached the trauma center and whether it was effective.

Doppler findings and results of any other investigative modalities employed in the peri-operative period, blood transfusions needed, reperfusion injury, presence or absence of acute kidney injury in the post-operative period. All cases were operated upon by a vascular surgeon, orthopaedic surgeon and general surgeons as a team. All patients were received in trauma centre where initial resuscitation, radiographs and hand held Doppler evaluation was done. Stable patients underwent color Doppler and/ or computed tomography angiography (CTA) and then shifted to the operating room (OR). Patients with active bleeding/hypovolemia or unstable to undergo investigations were directly shifted to OR for emergent exploration. External fixator application for displaced fracture segments of bone was resorted to, prior to undertaking vascular repair, in order to shorten time of surgery and to provide bony fixation requisite for the revascularisation procedure. Definitive orthopaedic management was planned for a later date once the patient stabilized. Standard principles of vascular surgery were undertaken.

Wound exploration was preceded by gaining proximal control of the injured artery followed by exploration of injured segment, evacuation of hematoma and securing hemostasis. Tourniquet was used in a few cases where there was delay in securing access due to difficult access of injured vessels. Arteries and veins with partial injury and no evident intimal injury proximally or distally were primarily repaired with cardiovascular expanded Polytetrafluoroethylene (ePTFE) sutures. The rest of arterial injuries were bypassed using contralateral great saphenous vein (GSV) leaving 4 to $5 \mathrm{~cm}$ of arterial segment proximal and distal to the injured segment. Venous injuries were managed by primary repair, reverse GSV (RGSV) bypass or ligation depending on the situation. Decision on venous repair/ligation depended upon length of venous injury, time elapsed since injury, availability of distal transected segment of vein and duration of surgery and general condition of the patient intra-operatively. Success of perfusion of the involved limb was confirmed on table 
using hand held Doppler. Two incision four compartment fasciotomy was done for lower limb arterial injuries which required more than 6 hours to restart limb perfusion from time of injury or in patients with evidence of nerve ischemia in the form of absence of active extension of great toe prior to surgery. Post-operatively, patients were kept on unfractionated heparin infusion with monitoring of activated partial thromboplastin time levels for therapeutic effect and later tapered to low molecular weight heparin once peri-operative bleeding concerns had abated. Prior to discharge from hospital, they were started on oral anti-platelet agent ecosprin. Patients with foot drop due to early nerve ischemia were managed with active physiotherapy in the post-operative period and continued long term. Early ambulation was instituted in most patients.

Treatment modality employed was noted in the Proforma with details of progress till discharge. Outcome measures that were observed were limb salvage, requirement of amputation and/or fasciotomy, time to ambulation, post treatment pressures/ABI, duration of hospitalization, morbidity and mortality.

\section{RESULTS}

There were a total of 46 cases and they were all males. The age range was 21 to 47 years. There were 39 arteries in-

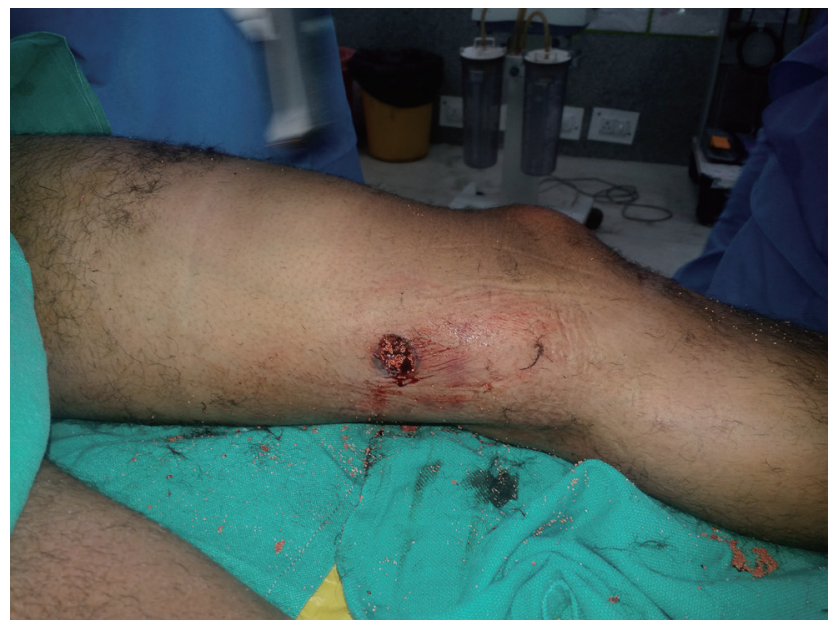

Fig. 1. Gun-shot wound thigh-entry wound. jured and a total of 26 venous injuries. Nineteen patients had both arterial and venous injuries. Total number of vascular injuries amounted to 65 vessels in 46 patients. Lower limb arteries were injured in 25 cases (Figs. 1-3), upper limb in 11 cases (Figs. 4, 5), one case of carotid artery injury and two cases of renal artery injury (Table 1). There were 17 cases of lower limb vein injuries, five cases of upper limb venous injuries, two cases of internal jugular vein (IJV) injury, and one case of renal vein and superior mesenteric vein injury, respectively (Table 2). Twenty-two out of 25 cases of lower limb arterial injury cases came to trauma centre with an effective tourniquet in place $(88 \%)$. Of the rest three cases, one was found to have transected thrombosed artery on exploration and there was no active bleeding on arrival while the other two were actively bleeding leading to large hematomas. A total of 42 cases presented within 12 hours of suffering

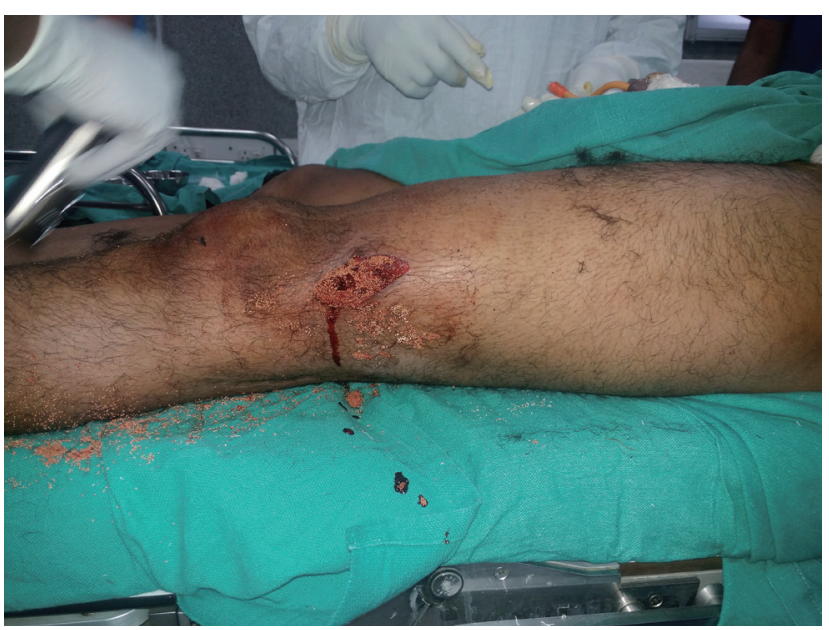

Fig. 2. Gun-shot wound thigh-exit wound.

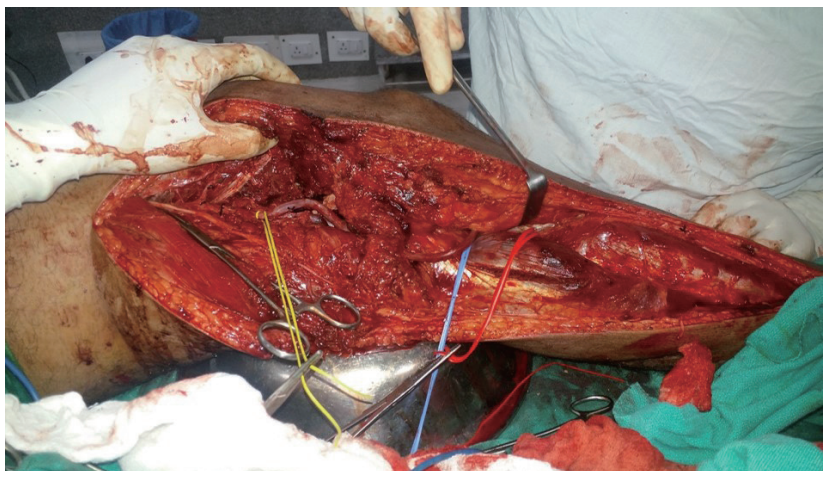

Fig. 3. Popliteal artery-revese great saphenous vein bypass. 


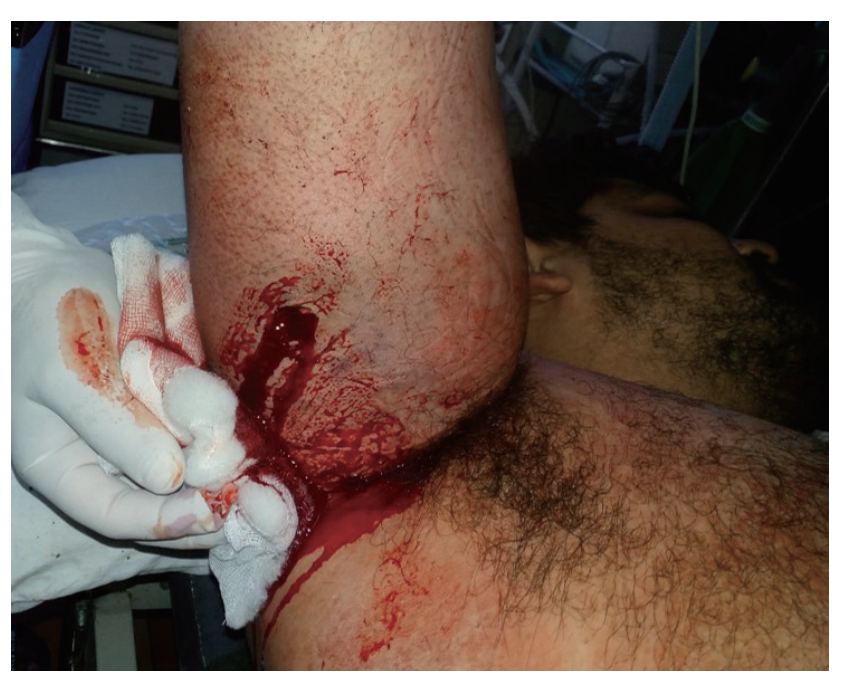

Fig. 4. Gun-shot wound right arm.

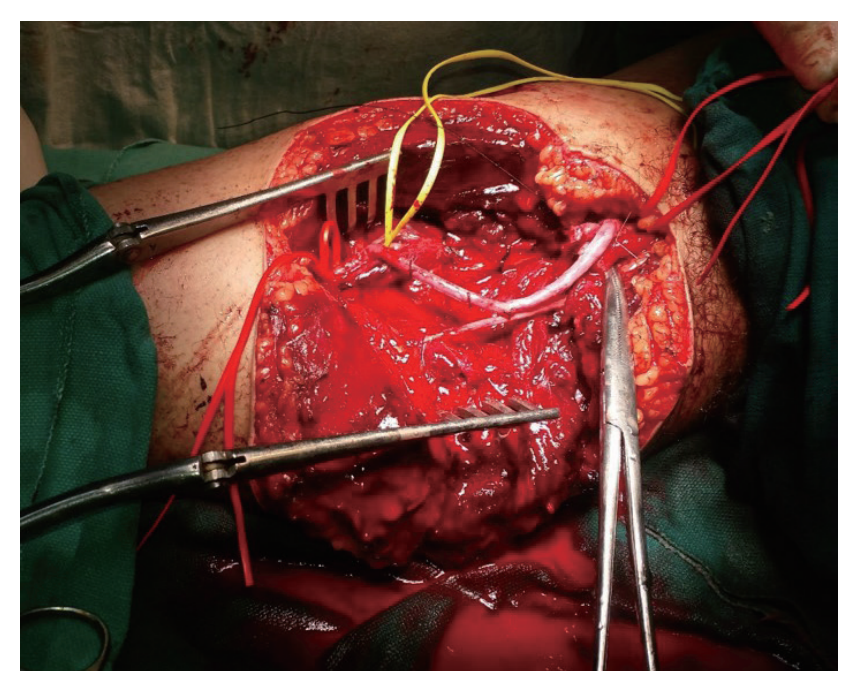

Fig. 5. Brachial artery bypass.

Table 1. Distribution of arterial injuries

\begin{tabular}{|c|c|c|c|c|}
\hline Ser No. & Artery injured & No. of patients & $\begin{array}{l}\text { No. of patients with } \\
\text { associated venous injury }\end{array}$ & Isolated arterial injuries \\
\hline 1 & Femoral artery & 6 & 2 & 4 \\
\hline 2 & Popliteal artery & 13 & 8 & 5 \\
\hline 3 & Tibial arteries-including ATA, PTA, Per & 6 & 3 & 3 \\
\hline 4 & Axillary artery & 1 & Nil & 1 \\
\hline 5 & Brachial artery & 5 & 3 & 2 \\
\hline 6 & Radial artery & 3 & 1 & 2 \\
\hline 7 & Ulnar artery & 2 & 1 & 1 \\
\hline 8 & Renal artery & 2 & 1 & 1 \\
\hline \multirow[t]{2}{*}{9} & Carotid artery & 1 & Nil & 1 \\
\hline & Total & 39 & 19 & 20 \\
\hline
\end{tabular}

No.: number, ATA: anterior tibial artery, PTA: posterior tibial artery, Per: peroneal artery.

Table 2. Distribution of venous injuries

\begin{tabular}{|llcl|}
\hline Ser No. & \multicolumn{1}{c|}{ Vein injured } & No. of patients & \multicolumn{1}{c|}{ Remarks } \\
\hline 1 & Internal Jugular vein & 2 & \\
2 & Superior mesenteric vein & 1 & Injured along with renal artery \\
3 & Renal vein & 1 & 2 cases of both fem art \& vein injury and 1 case of isolated fem vein injury \\
4 & Femoral vein & 3 & 8 cases of both pop art \& vein injury and 3 cases of isolated pop vein injury \\
5 & Popliteal vein & 11 & All 3 cases of art \& vein injuries \\
\hline 7 & Tibial veins & 3 & All 3 cases of brachial art \& basilic vein injuries \\
\hline 8 & Basilic vein & 3 & Both cases with either radial/ulnar artery injury \\
\hline
\end{tabular}

No.: number. 
the injury and complete arterial transections were found in 33 cases $(80.49 \%)$. There were three mortalities (mortality rate, $6.52 \%$ ). Two patients died due to acute kidney injury and multi organ failure as a complication of reperfusion injury and complications of massive transfusion, the third patient had suffered multiple gunshot wounds in the abdomen with splenic injury, left kidney injury and multiple bowel perforations along with left renal artery transection. He came in without pulse and was salvaged with splenectomy, nephrectomy, renal artery ligation and resection anastomosis of small bowel. He had required massive transfusion and died on 7th day after the injury due to multi-organ dysfunction syndrome and sepsis.

Three patients required major amputations (two transtibial and one tansfemoral) with an amputation rate of $8.33 \%$ (out of $36 \mathrm{limb}$ arterial injuries). Of those who presented within 12 hours of injury, there was only one amputation while all other limbs were salvaged with a limb salvage rate of $95.24 \%$. Of the patients who presented after 12 hours of injury, one was an IJV, two popliteal arteries and one peroneal artery injury. Both the cases of popliteal artery injuries reaching after 12 hours of injury needed trans-tibial amputations due to prolonged ischemia time. Overall limb salvage rate was $91.67 \%$. Associated injuries included hemothorax, lung lacerations, splenic injury, kidney injury, laryngeal injury, small bowel \& large bowel perforations and various limb and spine fractures along with splinter injuries to various parts of the body. Popliteal artery was the commonest artery to be injured as was the corresponding popliteal vein. The commonest associated orthopaedic injury was femur fracture.

Tourniquet for hemostasis was used in five cases due to difficult access to vessels. Thirty-five arteries required RGSV bypass and three arteries were repaired primarily. Balance three injured arteries were ligated, namely one each peroneal, renal and external carotid artery (Fig. 6). Thirty-three GSVs were harvested from the contralateral limb whereas two GSVs were harvested from the same limb due to injury in contralateral leg. Venous repairs were undertaken in 12 cases and ligation was done in 14 cases (Fig. 7). Eighteen patients required two incision four compartment fasciotomy (all cases of femoral and popliteal artery injuries) including one of forearm fasciotomy for a brachial artery injury with delayed presentation. Fifteen patients had foot drop, out of which four were transient and recovered in the early post-surgery period. The rest of the 11 patients were managed with active physiotherapy and nine recovered well and could ambulate without support on follow-up. Complications are outlined in Fig. 8. Poor prognostic markers for limb salvage were increasing time to present to the trauma centre, hypovolemic shock at presentation, multi-organ trauma and associated venous injuries.

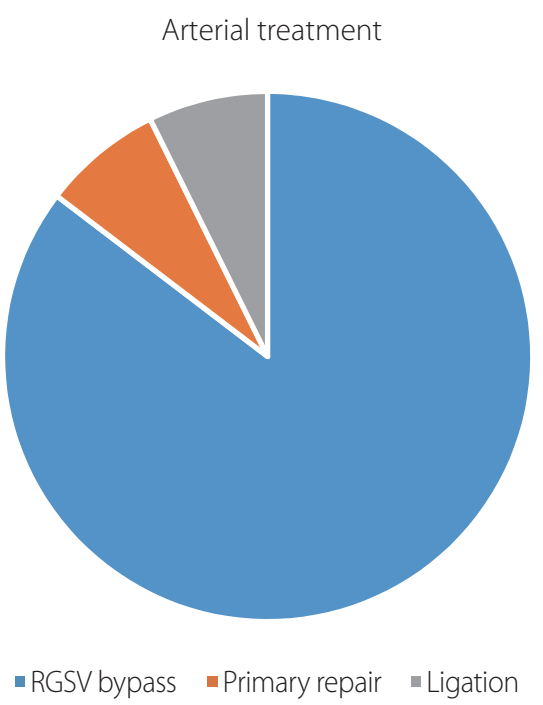

Fig. 6. Arterial injury treatment. RGSV: reverse great saphenous vein.
Venous treatment

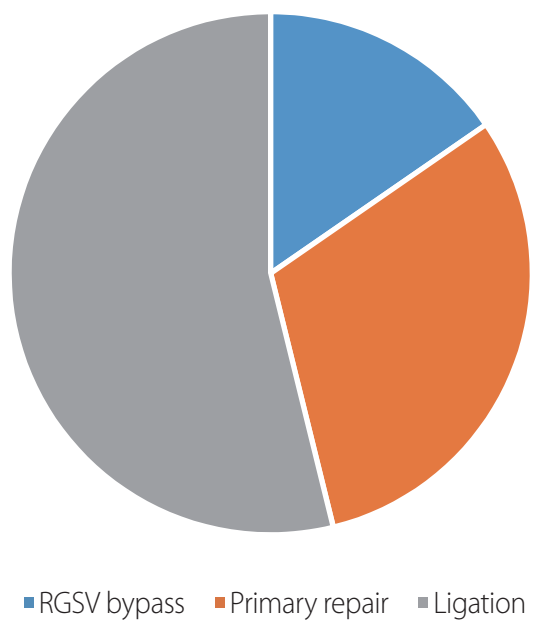

Fig. 7. Venous injury treatment. RGSV: reverse great saphenous vein. 


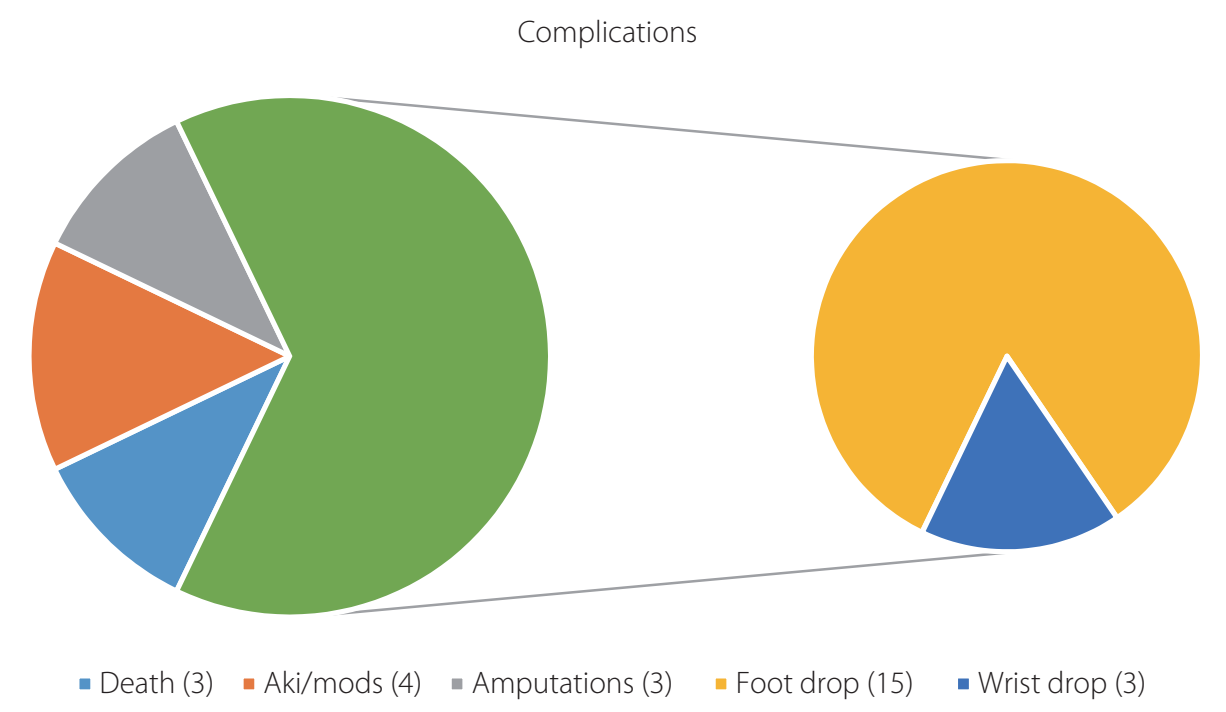

Complications

Fig. 8. Complications. AKI: acute kidney injury, MODS: multi-organ dysfunction syndrome.

\section{DISCUSSION}

The standard of care for managing vascular injury remained arterial and venous ligation until the 1950s, a technique that dated back to the 16th century. DeBakey and Simeone [2], in their classic work describing 2,471 vascular injuries sustained by American forces in World War II, stated that ligation was "not a procedure of choice. It is a procedure of stern necessity, for the purpose of controlling haemorrhage". They reported an amputation rate of $49 \%$. The amputation rate in the Korean War decreased to $13 \%$ among 227 cases of acute suture repair, thus confirming the benefit of routine repair of acute vascular injuries in a combat setting [3]. These results were identical to the ones from the Vietnam War probably due to rapid access to definitive care achieved by evacuation of casualties by helicopter within an average of 65 minutes [4]. Extremity vascular injuries predominate, representing 50 to $70 \%$ of all injuries treated during Operation Iraqi Freedom, and exsanguination from extremity wounds is the leading cause of preventable death on the modern battlefield [5]. Amputation rates following most civilian extremity vascular injury are quoted to be less than 5\% [6]. In our study, the rates of amputations from the variety of vascular trauma are $8.67 \%$ which are comparable to other studies. This reflects the delay in transfer of the patients to a trauma center or a tertiary care hospital for emergency surgery. Also, the nature of trauma being in anti-terrorist action involving blast injuries and gun-shot wounds differs from other studies reported in literature.

Existing literature suggests that peripheral vascular trauma typically occurs in males (72 to $90 \%$ ) between the ages of 20 and 40 years [7]. This study had $100 \%$ male patients and the age range was 21 to 47 years, which is comparable to other studies. The male preponderance is due to the nature of the security forces deployed in direct action against terrorist activities. The age range also reflects the active age group of the security forces that were treated in this study. The incidence of vascular injury among all forms of trauma is cited to be very low, ranging from $0.2 \%$ to $2 \%$ in military series, and up to $3.7 \%$ in civilian series $[8,9]$.

Studies suggest that early fasciotomy is associated with improved outcomes in patients with lower extremity vascular trauma treated with surgical intervention. Farber et al. [10] compared early fasciotomy $(<8$ hours after open vascular repair) with late fasciotomies ( $>8$ hours after vascular repair) in 612 patients undergoing vascular repair for traumatic vascular injuries. On multivariable analysis, early fasciotomy was associated with a four-fold lower risk of amputation. In our study, the late group was defined as the patients reaching hospital after 12 hours of injury. Both patients with popliteal artery injuries in the late group underwent amputation which is similar to reports 
in existing literature, suggesting that time to reaching hospital and vascular repair are central to the prognosis of salvage of the limb.

Popliteal vascular trauma remains a challenging entity, and carries the greatest risk of limb loss among the lower extremity vascular injuries. Sciaretta et al. [11] studied 47 patients with 78 traumatic popliteal vascular injuries and concluded that the risk for amputation is increased with longer intensive care unit stay and the use of PTFE grafting for vascular repair. Early restoration of blood perfusion, frequent use of interposition grafts with autogenous saphenous vein, and liberal use of fasciotomies play important role to achieve acceptable outcomes. In our study, popliteal vascular injuries lead to the maximum number of amputations. There were two amputations out of the 17 limbs that suffered popliteal vascular injuries amounting to $66.67 \%$ of all amputations in our study. Also, we refrained from using PTFE grafts and only RGSV was used for bypass grafting of arteries and veins.

There are only a few reports from the Indian subcontinent. Menakuru et al. [12] published a study on extremity vascular trauma in civil population in North India. They included 148 patients, however, only 16\% cases had suffered penetrating trauma and only seven patients had suffered gunshot wounds. It demonstrates the differences in mechanism of trauma in the civilian setting vis-avis anti-terrorism action suffered by security forces. The mortality rate in the study was $8 \%$, which is comparable to our death rate of $6.52 \%$. Further, the amputation rate of $6 \%$ is comparable to our rate of $8 \%$ and the difference could be due to the nature of trauma, blunt vis-a-vis high kinetic injuries in our setting.

Use of tourniquets in extremity vascular trauma plays a key role in saving lives by preventing exsanguination. Passos et al. [13] studied the use of tourniquets in the pre-hospital/traumav bay setting in a series of $190 \mathrm{pa}-$ tients suffering civilian extremity vascular trauma. They observed that only four patients had a tourniquet and six patients died of exsanguination for lack of a tourniquet. In our study, almost all patients (91.67\%) were brought with a tourniquet in place. This would have aided in improving the survival rate in our series. However, improperly placed tourniquets or ones placed for too long a time period would probably lead to loss of limb due to prolonged ischemia time.

Endovascular techniques have revolutionized the management of patient with vascular trauma. It is the newest weapon in the armamentarium of the vascular surgeon, providing similar outcomes with less morbidity to the patient. Acute care surgeons trained in endovascular techniques can safely treat traumatic vascular injury with good success [14]. Non-operative management is now common for non-occlusive injuries diagnosed on computed tomography arteriography [15]. The role of endovascular techniques like haemorrhage control with balloons, embolization (coils, plug, glue, etc.) and stenting/stent grafting in war/anti-terrorist trauma leading to penetrating injuries is still evolving and the lack of endovascular facilities at our centre precluded us from studying this option. However, studies should be undertaken to compare open surgery with endovascular techniques in the penetrating vascular trauma setting.

\section{CONCLUSION}

Vascular trauma accounts for $1 \%$ to $16 \%$ of all cases of trauma as per available literature. We outline the importance of attempting limb salvage even in cases with delayed presentation and appearing to be non-salvageable provided being done at a centre with vascular expertise. However, the threat to life should be given due consideration before attempting revascularisation and the decision should be individualized as per the case. A team approach with vascular surgeons, orthopaedic surgeons, general surgeons and critical care anaesthesiologists all aboard improve the outcomes manifold. Use of tourniquets and early fasciotomies has been emphasized which probably leads to a higher limb and life salvage rate. Bypassing arteries using native veins plays a central role in revascularisation in the setting of penetrating injuries to vessels. This is probably the largest study on penetrating vascular trauma in anti-terrorism ops from the Indian subcontinent and highlights the significance of prompt recognition, decision making and availability of vascular expertise in optimally managing cases of arterial and venous trauma. 


\section{REFERENCES}

1. He JC, Clancy K, Schechtman D, Conrad-Schnetz KJ, Claridge JA. Traumatic vascular injuries: who are repairing them and what are the outcomes? Am J Surg 2016;211:619-25.

2. DeBakey ME, Simeone FA. Battle injuries of the arteries in World War II: an analysis of 2,471 cases. Ann Surg 1946;123:53479.

3. Hughes CW. Arterial repair during the Korean War. Ann Surg 1958;147:555-61.

4. Rich NM, Baugh JH, Hughes CW. Acute arterial injuries in Vietnam: 1,000 cases. J Trauma 1970;10:359-69.

5. Starnes BW, Beekley AC, Sebesta JA, Andersen CA, Rush RM Jr. Extremity vascular injuries on the battlefield: tips for surgeons deploying to war. J Trauma 2006;60:432-42.

6. Diamond S, Gaspard D, Katz S. Vascular injuries to the extremities in a suburban trauma center. Am Surg 2003;69:848-51.

7. Mattox KL, Feliciano DV, Burch J, Beall AC Jr, Jordan GL Jr, De Bakey ME. Five thousand seven hundred sixty cardiovascular injuries in 4459 patients. Epidemiologic evolution 1958 to 1987. Ann Surg 1989;209:698.

8. Beebe GW, DeBakey ME. Battle casualties: incidence, mortality and logistic considerations. Illinois: Springfield and Charles C. Thomas publishers; 1952.

9. Oller DW, Rutledge R, Clancy T, Cunningham P, Thomason M,
Meredith W, et al. Vascular injuries in a rural state: a review of 978 patients from a state trauma registry. J Trauma 1992;32:7405; discussion 745-6.

10. Farber A, Tan TW, Hamburg NM, Kalish JA, Joglar F, Onigman $\mathrm{T}$, et al. Early fasciotomy in patients with extremity vascular injury is associated with decreased risk of adverse limb outcomes: a review of the National Trauma Data Bank. Injury 2012;43:1486-91.

11. Sciaretta JD, Macedo FI, Otero CA, Figueroa JN, Pizano LR, Namias N. Management of traumatic popliteal vascular injuries in a level I trauma center: a 6-year experience. Int J Surg 2015;18:136-41.

12. Menakuru SR, Behera A, Jindal R, Kaman L, Doley R, Venkatesan R. Extremity vascular trauma in civilian population: a seven-year review from North India. Injury 2005;36:400-6.

13. Passos E, Dingley B, Smith A, Engels PT, Ball CG, Faidi S, et al. Tourniquet use for peripheral vascular injuries in the civilian setting. Injury 2014;45:573-7.

14. Brenner M, Hoehn M, Teeter W, Stein D, Scalea T. Trading scalpels for sheaths: catheter-based treatment of vascular injury can be effectively performed by acute care surgeons trained in endovascular techniques. J Trauma Acute Care Surg 2016;80:7836.

15. Feliciano DV. For the patient-evolution in the management of vascular trauma. J Trauma Acute Care Surg 2017;83:1205-12. 